\title{
Errata: On the Metric Properties of the Feigenbaum Attractor $^{1}$
}

\section{Erik Aurell}

Received July 23, 1987

There is a consistent misprint beginning at Eq. (2.1), where $f p_{m+1}$ is written $f p m+1 . m+1$ is an index of the parameter $p$ as defined in the paragraph preceding Eq. (2.1), and should be read as such in this and similar formulas throughout.

Equation (A.2) should read

$$
\begin{aligned}
\sigma\left(j / 2^{m+1}+\right)-\sigma\left(j / 2^{m+1}\right) \\
=\sigma\left(j / 2^{m+1}\right)\left\{\frac{A\left(i_{0}, \ldots, i_{m}\right)+A\left(i_{0}^{\prime}, \ldots, i_{m}\right)}{A\left(i_{1}, \ldots, i_{m}\right)+A\left(i_{1}^{\prime}, \ldots, i_{m}\right)}-1\right\} \\
=\frac{\sigma\left(j / 2^{m+1}\right)}{A\left(i_{1}, \ldots, i_{m}\right)+A\left(i_{1}^{\prime}, \ldots, i_{m}\right)}\left\{\left[A\left(i_{0}, \ldots, i_{m}\right)-A\left(i_{1}, \ldots, i_{m}\right)\right]\right. \\
\left.\quad+\left[A\left(i_{0}^{\prime}, \ldots, i_{m}\right)-A\left(i_{1}^{\prime}, \ldots, i_{m}\right)\right]\right\}
\end{aligned}
$$

\footnotetext{
${ }^{1}$ This paper appeared in J. Stat. Phys. 47:439 (1987).
} 